\title{
La realidad aumentada como tecnología emergente para la innovación educativa
}

\author{
Noelia Margarita Moreno Martínez \\ Universidad de Málaga \\ Juan J. Leiva Olivencia. \\ Universidad de Málaga \\ Eloy Lopez Meneses. \\ Universidad Pablo de Olavide (Sevilla)
}

\begin{abstract}
Resumen: En la actualidad la tecnología basada en la realidad aumentada ha comenzado ha mostrarnos sus enormes beneficios en el ámbito educativo. Su implementación en los diferentes niveles y disciplinas se hace posible gracias a la gran variedad de aplicaciones y programas de realidad aumentada que se están diseñando con un carácter didáctico y pedagógico cuyos contenidos de aprendizaje se muestran en modalidad virtual para complementar el contexto real y enriquecerlo. De este modo, desde un paradigma constructivista, el discente contando con el apoyo y orientación por parte del docente, se convierte en creador y constructor activo de escenarios de aprendizaje aumentados. Y por otro lado, desde un modelo conectivista de aprendizaje en red tiene lugar una actitud 2.0 basada en compartir, construir e intercambiar recursos digitales poniéndose en marcha la inteligencia colectiva al servicio del conocimiento y enriquecimiento de la comunidad educativa. En el presente trabajo se realiza una revisión y un análisis, desde un punto de vista epistemológico, del concepto de Realidad Aumentada; una reflexión acerca del tipo de aprendizaje híbrido que generan los entornos de Realidad Aumentada; y se finaliza con la presentación y descripción de herramientas para la creación de escenarios de realidad aumentada en el aula.

Palabras clave: Realidad Aumentada; Tecnología Emergente; Aprendizaje Híbrido; Innovación Educativa.

Abstract: Augmented-reality-based technology has currently begun to show us its huge benefits in the field of education. Its implementation at different levels and disciplines becomes possible thanks to the great variety of augmented reality applications and programs that are being designed for didactic and pedagogical purposes, the learning contents of which are shown in a virtual format seeking to complement the actual context and to enrich it. In this way, on the basis of a constructivist paradigm, the student can take advantage of the teacher's support and guidance to become an active creator and builder of augmented learning scenarios. To which must be added that a connectivist networked learning model favours a 2.0 attitude based on sharing, building and exchanging digital resources, thus implementing collective intelligence at the service of the educational community's knowledge and enrichment. The present work offers a review and an analysis about the concept of Augmented Reality from an epistemological point of view; a reflection on the type of hybrid learning that is generated by augmented reality environments; and it finishes with the presentation and description of tools for the creation of augmented reality scenarios in the classroom.
\end{abstract}

Keywords: Augmented Reality; Emergent Technology; Hybrid Learning; Educational Innovation.

\section{Realidad Aumentada: aclaración conceptual}

Como exponen Reig (2012 y 2013), así como Reig y Vílchez (2013), son muchas las instancias que prevén el aumento del uso y la incorporación de dispositivos y aplicaciones en el ámbito educativo como factor de calidad con un carácter innovador. Entre ellos cabe destacar los informes Horizon Report (2012, 2013, 2014 y 2015) liderados por el New Media Consortium y Educause con la colaboración de especialistas a nivel mundial y que constituye un proyecto de investigación de una década de duración diseñado para identificar y describir las 
tecnologías emergentes que puedan tener un impacto en el aprendizaje, la enseñanza y la investigación en el presente, futuro inmediato y futuro lejano de distintos países. Ya en su novena edición de la versión Internacional se destacaba especialmente el Mobile Learning, surgiendo a la vez temas asociados a éste, como son los libros electrónicos, el aprendizaje basado en juegos (Serious Game y Gamificación) o la Realidad Aumentada.

La Realidad Aumentada (RA en adelante) hace referencia a la visualización directa o indirecta de elementos del mundo real combinados (o aumentados) con elementos virtuales generados por un ordenador, cuya fusión da lugar a una realidad mixta (Cobo y Moravec, 2011, p. 105). En la misma línea Azuma (1997)), la concibe como aquella tecnología que combina elementos reales y virtuales, creando escenarios interactivos, en tiempo real y registrados en 3D. También es definida por Cabero (2013), Gómez (2013), Cabero y García (2016) como aquel entorno en el que tiene lugar la integración de lo virtual y lo real. Otros autores elaboran conceptos más complejos abarcando más elementos implicados en este proceso, como De Pedro (2011) el cual entiende la RA como aquella tecnología capaz de complementar la percepción e interacción con el mundo real, brindando al usuario un escenario real aumentado con información adicional generada por ordenador. De este modo, la realidad física se combina con elementos virtuales disponiéndose de una realidad mixta en tiempo real.

Kato (2010) define la RA como objetos virtuales o anotaciones que pueden ser superpuestos en el mundo real como si realmente existieran. Por su parte, Basogain, Olabe, Espinosa, Rouèche y Olabe (2007) afirman que:

"la RA no reemplaza el mundo real por uno virtual, sino al contrario, mantiene el mundo real que ve el usuario complementándolo con información virtual superpuesto al real, de ese modo, el usuario nunca pierde el contacto con el mundo real que tiene al alcance de su vista y al mismo tiempo puede interactuar con la información virtual superpuesta” (p.1).

Así pues, tras una revisión conceptual de dicha tecnología, nosotros podríamos aportar la siguiente definición desde una perspectiva de aplicabilidad educativa considerándola como aquella tecnología emergente que nos permite crear entornos de enseñanza-aprendizaje mixtos donde se combinan elementos virtuales y reales, sin confundirla con la realidad virtual, la cual, a diferencias de la anterior, sustituye por completo el mundo real por otro virtual constituyendo entornos de simulación. La RA trasladada al ámbito educativo, consiste en incorporar al contexto real de aprendizaje en el aula elementos $\mathrm{u}$ objetos virtuales tridimensionales o información digital adicional generados a través de dispositivos (móviles, tablet, gafas de RA, etc.) con el objetivo de complementar, reforzar, potenciar, amplificar y enriquecer los escenarios. De este modo, logramos aumentar las posibilidades de aprendizaje ya que el discente recibe más estímulos, favoreciendo, a su vez, no sólo el aprendizaje de contenidos, sino el desarrollo de la creatividad del alumnado y su interés por investigar y explorar para construir su conocimiento, dado el gran carácter motivador que incorpora esta herramienta. Pues Como dice Reinoso (2012, p. 371): "numerosas han sido las investigaciones que sugieren que la $R A$ refuerza el aprendizaje e incrementa la motivación por aprender". Por lo tanto, consideramos que las aplicaciones basadas en la realidad aumentada favorecen el aprendizaje por descubrimiento, mejoran la información disponible para los estudiantes ofreciendo la posibilidad de visitar lugares históricos y estudiar objetos muy difíciles de conseguir en la realidad. Este entorno 
permite que los discentes realicen su trabajo de campo, interactuando con los elementos generados de forma virtual.

Además, un elemento clave de la configuración de la RA como factor de innovación docente lo encontramos en lo que sería la construcción emergente de una Educación Personalizada, esto es, una educación que pretende dar respuesta a las necesidades personales de aprendizaje del alumnado con calidad educativa, $\mathrm{y}$, a la vez, fomentar un estilo híbrido de aprendizaje que podemos considerar como más avanzado y creativo. Y esto ocurre como consecuencia de una socialización digital que está cambiando la forma en que los jóvenes aprenden. Por ejemplo, durante mucho tiempo la pedagogía no ha considerado los videojuegos como una herramienta de interés científico, únicamente se le atribuía un carácter meramente lúdico y motivador para un aprendizaje teóricamente más laxo, e incluso se le concebía como un elemento distractor de un aprendizaje relevante. Hoy en día, los estudios más recientes transforman la pedagogía en una disciplina más dinámica, preocupada por cómo los nuevos cambios tecnológicos están generando nuevas y diversas formas de enseñar y aprender (Yuen, Yaoyuneyong y Johnson, 2013).

\section{2. ¿Qué características de aprendizaje híbrido nos aporta la RA?}

En primer lugar, la RA aporta inmediatez e interactividad intuitiva en el aprendizaje del alumnado. Esto implica que la realización de actividades de enseñanza-aprendizaje con RA son eminentemente prácticas. Así, el papel del docente no implica "desembuchar" ningún tipo de explicación magistral, ni tampoco nos interesa que el alumnado memorice conceptos o redes de conocimiento sin ningún tipo de comprensión. Lo que interesa con estas nuevas actividades educativas con RA son la inmediatez, la rápida asimilación de procedimientos de aprendizaje, el incremento de la motivación intrínseca y la búsqueda de respuestas pedagógicas múltiples y creativas (Di Serio, Ibáñez y Kloos, 2013).

En segundo lugar, la RA se caracteriza por un proceso de desarrollo cognitivo divergente, esto es, se aprende haciendo y planteando a la vez distintas fórmulas o caminos para la resolución de problemas prácticos.

En tercer lugar, el aprendizaje híbrido supone una reconceptualización de los roles del docente y discente, de tal forma que la RA subraya el carácter transfigurador de los papeles de quien enseña y aprende en una clase. Las clases son espacios de encuentro educativo y los docentes son formadores que aprenden mientras enseñan, y los alumnos aprenden a aprender y a enseñar.

En cuarto lugar, hay que señalar que la RA supone que cualquier elemento didáctico debe entenderse como pertinente en la medida en que responde a las demandas de necesidades personales de aprendizaje. Nos referimos al carácter multifacético de los recursos didácticos. No se trata de hacer una única actividad a partir de un enunciado o un material concreto, sea virtual o presencial, textual o audiovisual, sino que éste ofrezca diversas opciones de elaboración pedagógica. Obviamente, esto debe ser asumido por los docentes quienes deben ser orientadores y facilitadores de aprendizaje, sugiriendo y nunca imponiendo formas de resolución de problemas.

En quinto lugar, la RA es, en sí mismo, una forma global de enseñar y de aprender, por lo que su carácter globalizador y holístico supone un cambio de mirada en la tradicional forma fragmentada de enseñar y de aprender. Así, un estilo de aprendizaje híbrido únicamente se desarrolla en un contexto de aprendizaje en el que las actividades tienen un sentido continuo de desarrollo. Esa idea de continuum. Por ejemplo, científicos de la Universidad Carlos III de Madrid (UC3M) que han 
desarrollado un primer prototipo que demuestra el funcionamiento de una plataforma de este tipo. Así, Estos investigadores han empleado una plataforma de código abierto para la creación de mundos virtuales tridimensionales distribuidos (OpenWonderland) y aprovechan la geolocalización con la que ya cuentan los smartphones. El propósito es aprovechar las características de inmersión que brindan los entornos virtuales y la interactividad entre estos tres espacios (la realidad, el entorno virtual y el híbrido) para la creación de actividades e-learning en el campo de los idiomas.

Lo más importante de esta experiencia es el carácter global del aprendizaje en la virtualidad aumentada, así como la idea de obtener una interactividad significativa. Además, la ventajas de la aplicación de este tipo de tecnologías a este ámbito del $e$ learning son muy diversas. Como hemos mencionado con anterioridad, se espera que los alumnos estén más motivados para participar en el proceso de aprendizaje, al tratarse de actividades más interactivas y en las que el alumno puede experimentar diversas situaciones. Por otro lado, son espacios que pueden ser vistos como laboratorios donde las variables pueden estar más y mejor controladas por los pedagogos.

En definitiva, la búsqueda de escenarios más interactivos de enseñanza y aprendizaje están en la raíz y esencia del proceso educativo con RA (Dunleavy y Dede, 2014), donde es muy importante que el aprendizaje híbrido se pueda contextualizar y se puedan atravesar y solucionar problemas y necesidades emergentes de aprendizaje de forma reflexiva y creativa.

\section{Herramientas para la creación de escenarios de realidad aumentada en el aula}

A continuación, presentamos una serie de herramientas basadas en la tecnología de RA (tabla 1), la cual puede aplicarse en los diferentes niveles del sistema educativo obteniéndose resultados positivos para la adquisición de aprendizajes significativos en las diferentes áreas didácticas (Cabero, Leiva, Moreno, Barroso y López, 2016).

\begin{tabular}{|c|c|c|c|c|}
\hline Aplicaciones móviles & $\begin{array}{l}\text { Programas } \\
\text { para PC }\end{array}$ & $\begin{array}{l}\text { Plataformas } \\
\text { web }\end{array}$ & $\begin{array}{l}\text { Programas de } \\
\text { diseño gráfico y } \\
\text { modelado }\end{array}$ & $\begin{array}{l}\text { Galerías con } \\
\text { modelos 3D } \\
\text { (formatos .dae, } \\
\text {.obj, .fbx o .3ds) }\end{array}$ \\
\hline $\begin{array}{l}\text { - Aurasma } \\
\text {-Aumentaty Viewer } \\
\text { - Layar } \\
\text { - Quiver } \\
\text { - Chromville } \\
\text { - Chromville Barcy } \\
\text {-Chromville Science } \\
\text { - AR ARKids } \\
\text {-ARFlashcards Animal } \\
\text { Alphabet } \\
\text {-ARFlashcard Space } \\
\text { - AR Dino Roar } \\
\text { - AR DinoPark } \\
\text { - AnimalCAm } \\
\text { - Zookazam } \\
\text { - Arloon Anatomy } \\
\text { - Anatomy 4D } \\
\text { - The Brain } \\
\text { - AR iSkull } \\
\text { - AR Durolane } \\
\text { - FaceYou } \\
\text { - Taggar } \\
\text { - Wikitude } \\
\text { - Junaio }\end{array}$ & $\begin{array}{l}\text {-Aumentaty } \\
\text { Autor } \\
\text { - BuildRA } \\
\text { - EstARteco }\end{array}$ & $\begin{array}{l}\text {-Studio } \\
\text { Aurasma } \\
\text { - ARCrowd } \\
\text { - LearnAR } \\
\text { - Bakia } \\
\text {-Layar } \\
\text { Creator }\end{array}$ & $\begin{array}{l}\text {-SketchUp } \\
\text {-123D Design } \\
\text { - Creationist 3D } \\
\text { - Catch beta } \\
\text { - Blender }\end{array}$ & 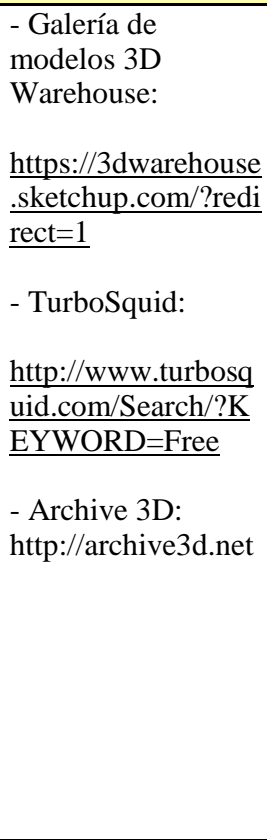 \\
\hline
\end{tabular}


\begin{tabular}{|l|l|l|l|}
\hline - Goggles & & & \\
- Aumentaty & & & \\
VSearch & & \\
- Sky Map & & \\
- Mapa estelar 3D & & \\
- Compass & & \\
-AR La Patena de & & & \\
Cástulo RA & & & \\
- AR Showcase & & & \\
- Visuar & & & \\
- EspiraRA & & & \\
\hline
\end{tabular}

Tabla 1. Herramientas de RA, programas de modelado y galerías para obtener modelos 3D en diferentes formatos

\subsection{Aplicaciones móviles de realidad aumentada}

Aurasma: Es una aplicación móvil multiplataforma que permite crear de forma sencilla y rápida escenarios de RA a partir de cualquier fotografía y que actuará como marcador. La aplicación nos ofrece una amplia galería de objetos virtuales y tridimensionales animados, aunque nos posibilita utilizar cualquier fotografía, imagen $\mathrm{u}$ objeto del mundo real, lo que permite aumentar cualquier cosa sin necesidad de imprimir ningún marcador o imagen. Además, crea en pocos minutos escenas de RA añadiendo capas virtuales de imagen, vídeo, animaciones o modelos $3 \mathrm{D}$, con posibilidad de compartirlas públicamente al instante. De esta forma, cualquier persona, siguiendo el canal público de la aplicación, podrá ver desde su dispositivo móvil las «auras» o escenas de RA. Inclusive, esas auras o imágenes creadas, se pueden geolocalizar, de tal forma que aunque fuesen públicas sólo se verían desde una zona geográfica determinada. Los creadores de la aplicación han puesto a disposición de los usuarios una plataforma web (Studio Aurasma) a través de la cual se puede realizar una mayor variedad de acciones desde la aplicación web, como crear auras con modelos 3D para posteriormente importarlas al móvil. La figura 1 se muestra un ejemplo. Inclusive edita las auras ya creadas y añade más de una capa virtual a una imagen. Más información: http://www.aurasma.com

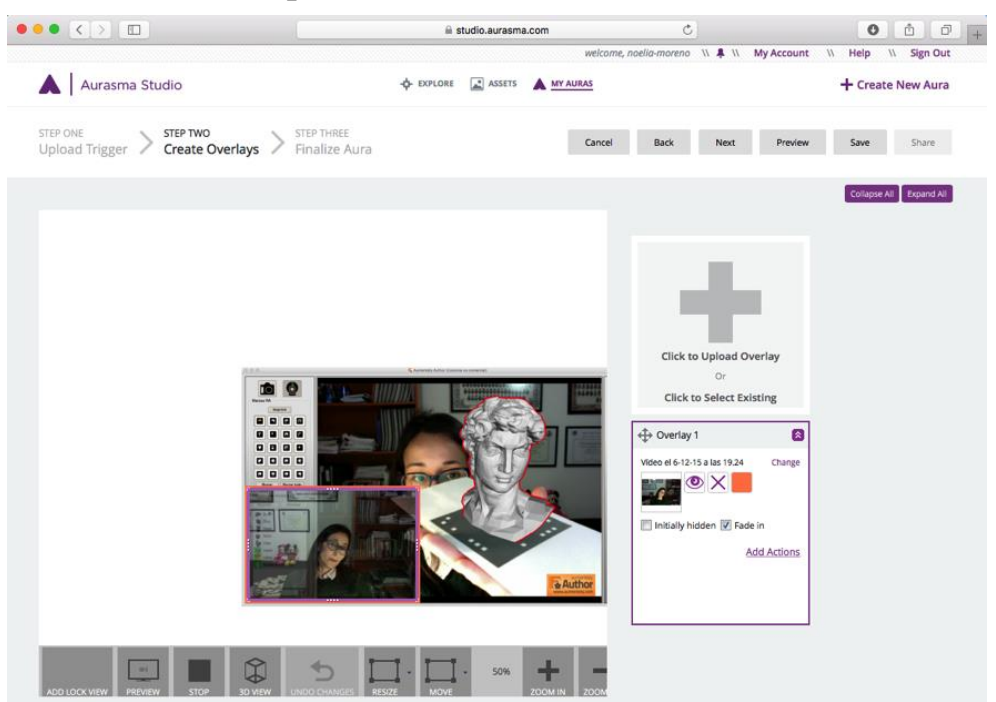

Figura 1. Plataforma Aurasma Studio para la configuración y diseño de contextos de realidad aumentada. Augment: Aplicación que permite crear entornos aumentados mediante el marcador oficial de Augment, disponible en la web: http://www.augment.com/ es/trackers/; o bien creando nuestro propio marcador a partir del cual se despliega un elemento virtual en 3D. Tras previo registro en la plataforma web Augment: http://www.augment.com/es/, se puede emplear cualquier archivo 3D en formato .dae, .obj, .fbx o .3ds, que podemos exportar desde el programa SketchUp o galerías de 
modelos tridimensionales como 3D Warehouse y Archive 3D. Posteriormente podemos subirlos a nuestra plataforma Augment en formato .zip para insertarlos en el contexto real y crear un escenario mixto aumentado usando la aplicación móvil Augment. Las figuras 2 y 3 muestran ejemplos de escenarios de realidad aumentada para el análisis y estudio de contenido didáctico diverso.

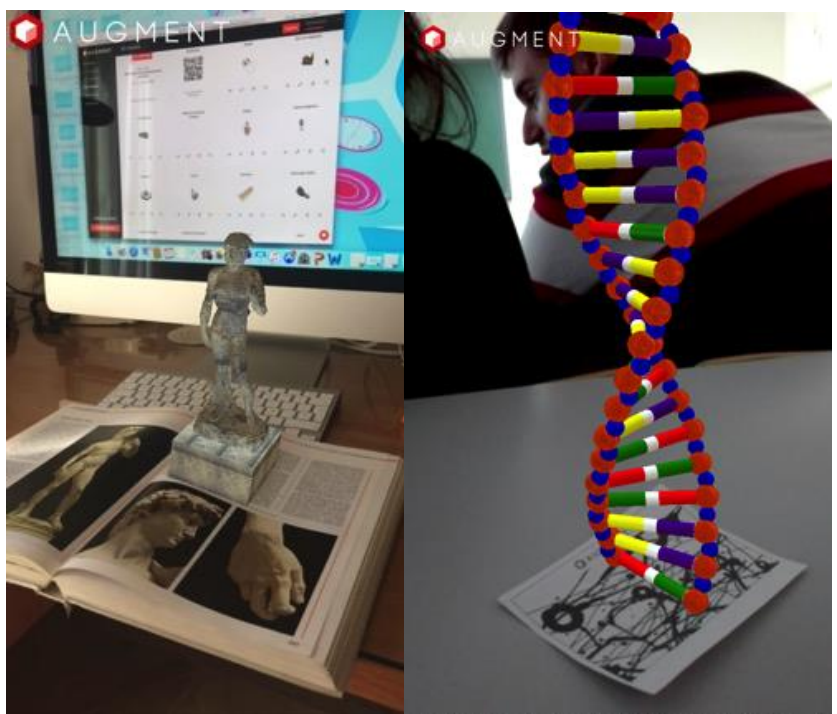

Figura 2 y 3 . Muestra de escenarios de realidad aumentada con contenidos didácticos.

Aumentaty Viewer: Es un visualizador de proyectos de realidad aumentada con ficheros en formato .obj realizados con el programa de ordenador Aumentaty Author. Más información: http://author.aumentaty.com/acerca-de-aumentatyauthor?qt-info=2

Quiver: Aplicación basada en la RA y en el mundo virtual que posibilita colorear láminas impresas que se obtienen de la web (http://quivervision.com/es/) para, posteriormente, utilizar la cámara de móvil y lograr que adquieran que los escenarios de las láminas y los elementos que lo conforman cobre vida, ofreciendo al alumnado un entorno enriquecido y dinámico para estimular su creatividad y el aprendizaje basado en el descubrimiento y la exploración en nuevos escenarios digitales. Las figuras 4, 5 y 6 muestran láminas con contenidos educativos que se transforman en modelos tridimensionales para su estudio y análisis, incorporando actividades interactivas y gamificación.

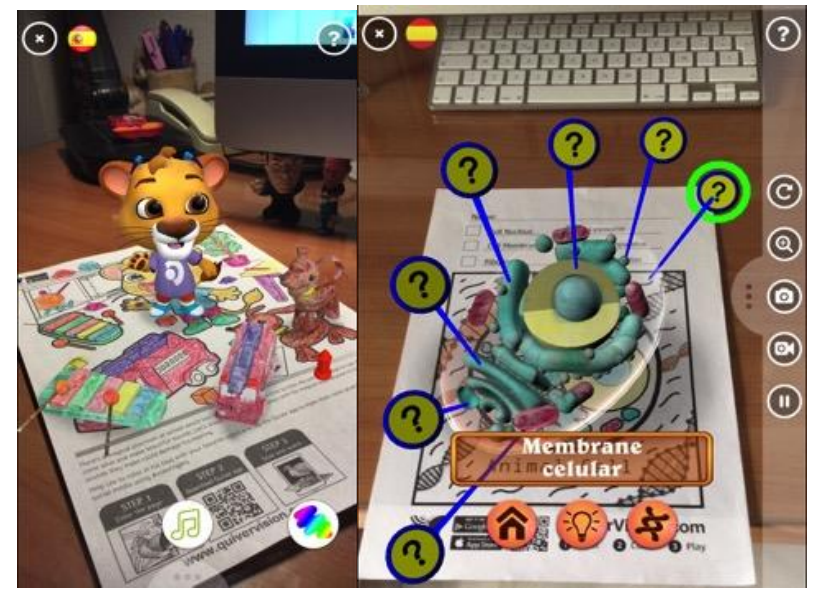




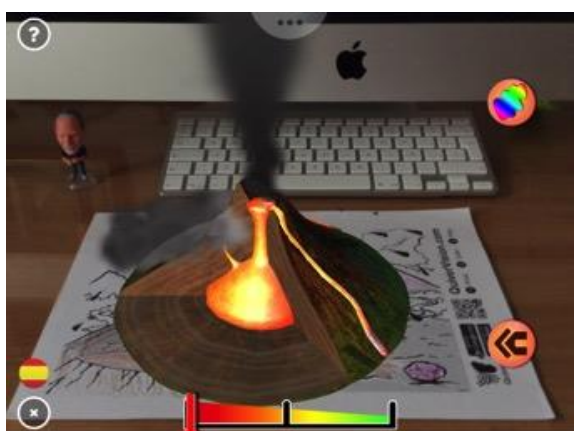

Figuras 4, 5 y 6. Ejemplos de láminas para la creación de escenarios de realidad aumentada con la aplicación móvil Quiver.

Chromville: Es una aplicación en la misma línea que la anterior, basada en láminas para colorear que actúan de marcadores para generar entornos de realidad aumentada. También existen otras aplicaciones de los mismos desarrolladores denominadas chromville Barcy, destinadas al abordaje del concepto del agua, energías renovables y chromville Science, para introducir al alumnado en experiencias. Las láminas impresas para colorear que actúan como marcadores para la creación de entornos de fantasía aumentada a través de la cámara del dispositivo, se obtienen a través de esta web: https://chromville.com. La figura 7 muestra un ejemplo.

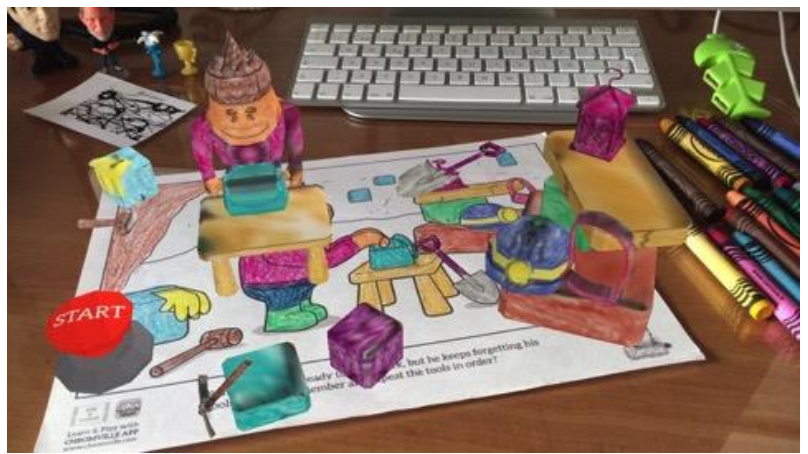

Figura 7. Escenario de realidad aumentado creado con la aplicación móvil Chromville.

AR ARKids: Es una aplicación que también nos permite atribuirle vida a los personajes de láminas impresas que podemos obtener de la siguiente web: http://www.ipad-kids.ru/ar-en.php

AR Flashcards Animal Alphabet: Es una aplicación por medio de la cual ofrecemos un escenario de aprendizaje del alfabeto, vocabulario de animales en inglés y diferentes especies de dinosaurios. Más información: http://arflashcards.com/. En las figuras 8 y 9 se muestran ejemplos.

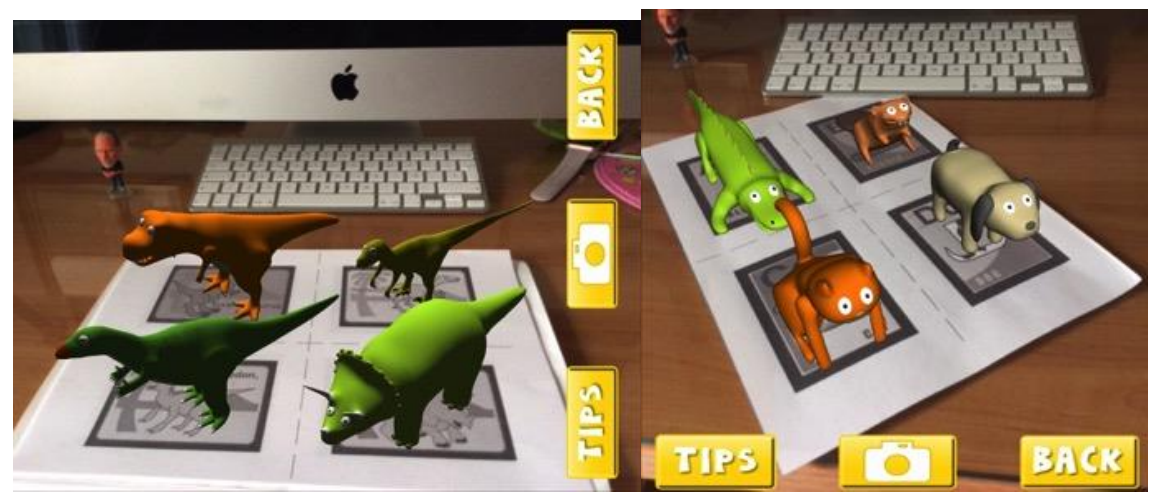

Figuras 8 y 9. Ejemplos de modelos tridimensionales generados con la herramienta de realidad aumentada AR Flashcards Animal Alphabet. 
AR Flashcard Space: Es una aplicación del mismo desarrollador que el anterior para la visualización de los planetas del sistema. Más información: http://arflashcards.com/ Las figuras 10 y 11 muestran ejemplos.

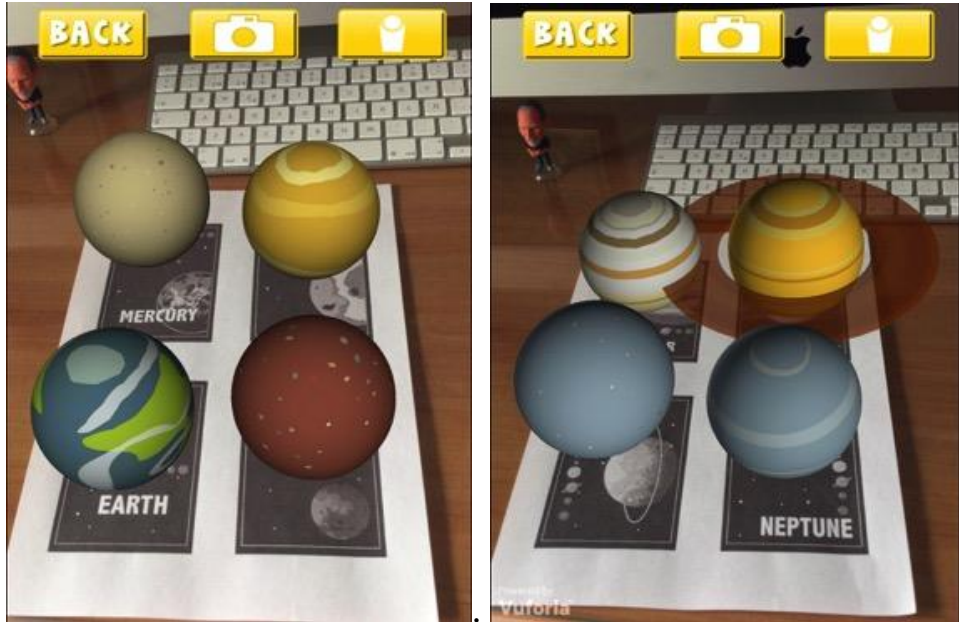

Figuras 10 y 11. Visualización de diferencias planetas tridimensionales mediante la aplicación móvil AR Flashcard Space.

Zookazam: a través de esta aplicación podemos añadir un amplio repertorio de animales de diversas especies en nuestro entorno real para conocer sus características. Más información acerca de esta aplicación: http://www.zookazam.com Las figuras 12 y 13 muestran ejemplos de contextos generados con tecnología de realidad aumentada.

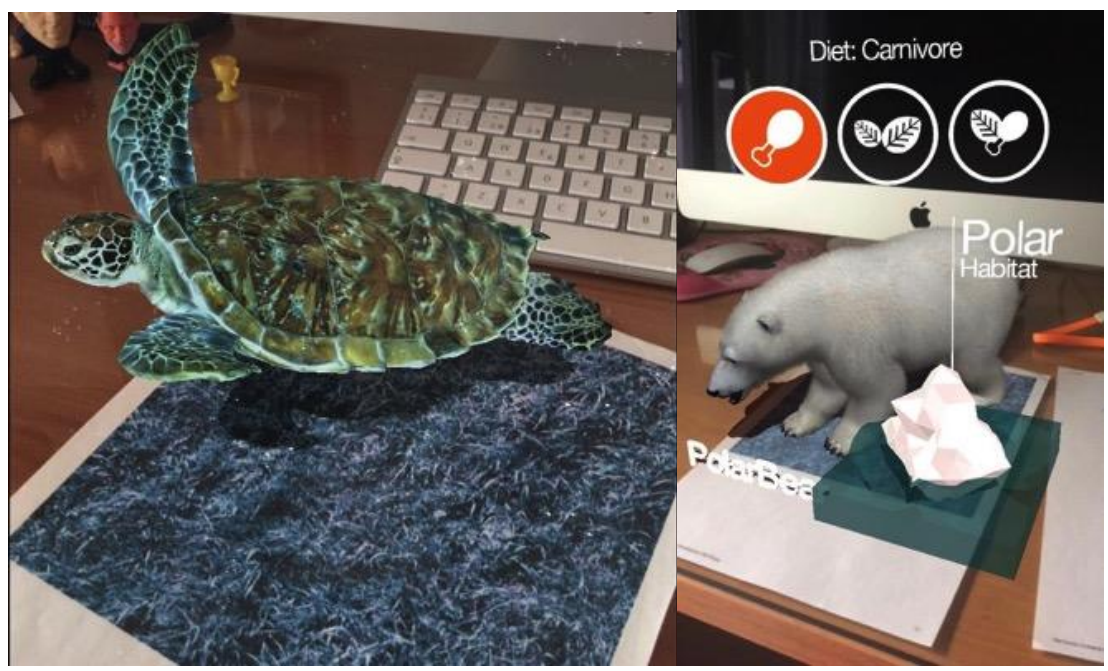

Figura 12 y 13. Animales tridimensionales insertados en el contexto real haciendo uso de la aplicación móvil de realidad aumentada ZooKazam.

AR Dino Roar: Nos permite crear un escenario de realidad aumentada insertando en el contexto real diversas especies de dinosaurios a partir de portadas o páginas de libros, revistas, periódicos que actúan como marcadores de los cuales surge el modelo 3D del dinosaurio.

Animal Cam y ARDinopark: Posibilita insertar modelos 3D virtuales de dinosaurios y otros animales animados en nuestro entorno real sin necesidad de marcador ya que incorpora un cuadro de mandos para posicionar el objeto $3 \mathrm{D}$ en el lugar deseado. 
Anatomy Arloon: Esta aplicación nos permite visualizar y analizar diferentes aparatos del cuerpo humano con máximo detalle. Además nos ofrece una sección de preguntas para consolidar los aprendizajes y explicaciones complementarias que describen el funcionamiento de los órganos internos del cuerpo humano. Las figuras 14 y 15, muestran capturas de pantalla del panel con las opciones para elegir el sistema objeto de estudio y un ejemplo en el que se inserta un modelo 3D del sistema muscular en el contexto real.

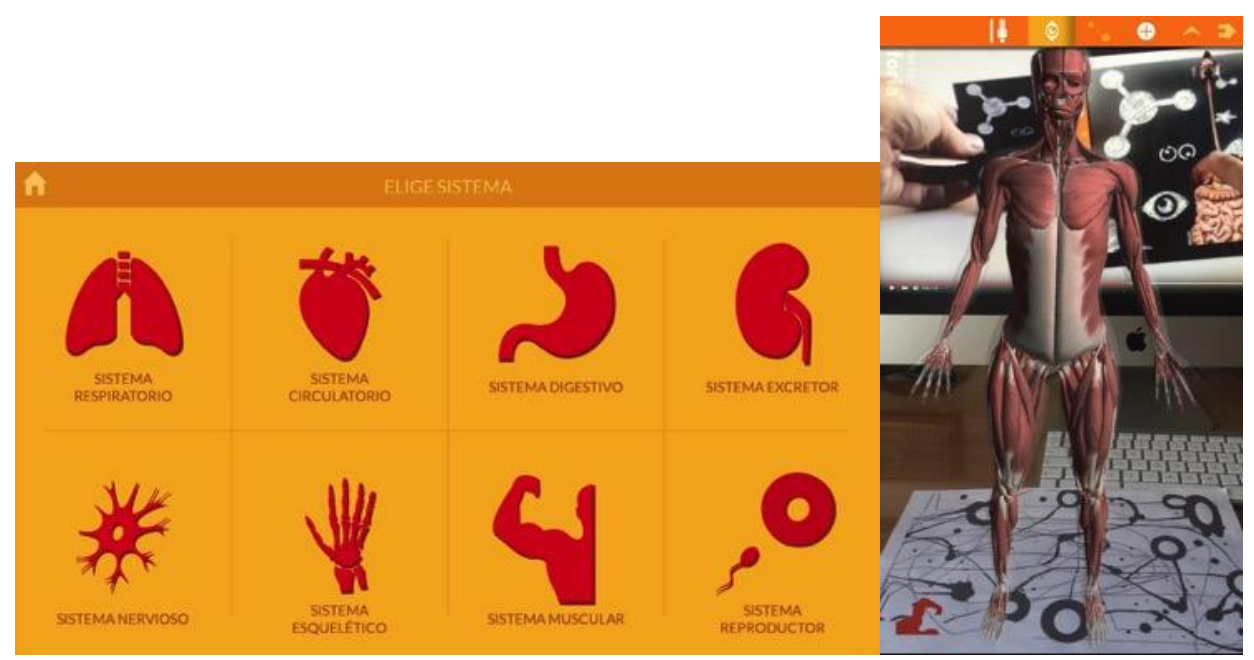

Figuras 14 y 15. Panel para elegir un sistema y muestra del sistema muscular.

Anatomy 4D: Nos permite la visualización de los diferentes aparatos, órganos y sistemas a través de una lámina del cuerpo humano y otra lámina del corazón, las cuales actúan como marcadores para generar el escenario de aprendizaje aumentado. Dichas láminas las podemos descargar de la siguiente web: http://blog.daqri.com/anatomy- 4d-changes-the-way-we-learn-about-the-human-body Las figuras 16 y 17 muestran los ejemplos de visualización de los modelos 3D a través de la aplicación móvil.

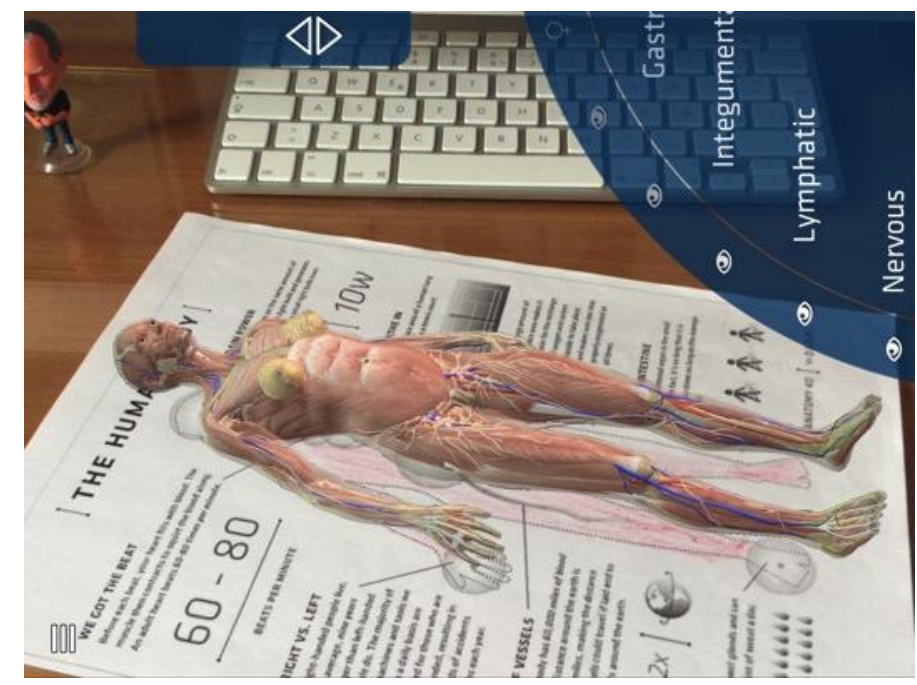




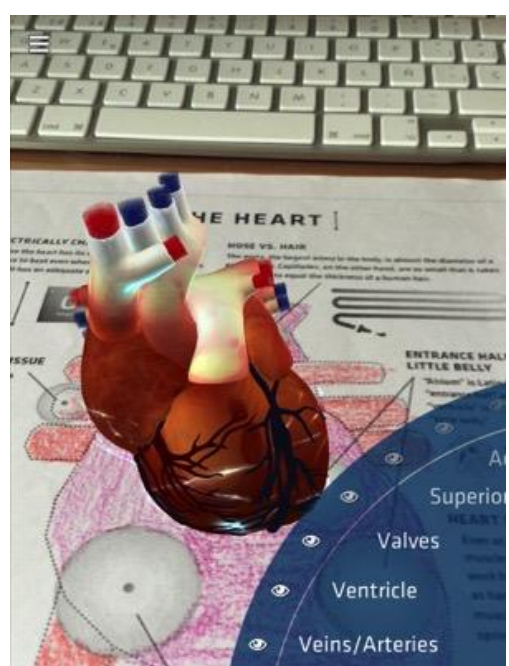

Figuras 16 y 17. Visualización del cuerpo humano y el corazón a través de la aplicación móvil Anatomy 4D.

The Brain AR: Se emplea para el estudio del sistema muscular, el sistema respiratorio, el sistema circulatorio, el sistema esquelético, el cerebro y las neuronas y sus conexiones.

iSkull AR: Está indicada para el estudio de todos los huesos del cráneo. Los estudiantes de anatomía pueden imprimir el marcador de la siguiente web: http://www.mahei.es/iskull.php?lang=es para su posterior escaneo y visualización con la aplicación.

Durolane: Esta aplicación supone un recurso complementario del resto de materiales empleados en el aula para el estudio de modelos anatómicos en 3D de una rodilla y una cadera. Mediante tecnología de realidad aumentada se ilustra con total realismo el funcionamiento de las articulaciones, sus diferentes secciones, la localización de las distintas patologías y su tratamiento, así como el proceso a seguir en caso de artroscopia o infiltración. Incluso ofrece un entorno de simulación del proceso de infiltración del ácido hialurónico para el tratamiento de la artrosis. Para poder visualizar correctamente los modelos en 3D es necesario descargar e imprimir un marcador del siguiente enlace: http:// bit.ly/1HqDZgC.

FaceYou: Por medio de fotos o vídeos, podemos adoptar el rostro que queramos mediante tecnología basa- da en inteligencia artificial y realidad aumentada. Esta aplicación se originó a partir de una demostración técnica creada por investigadores de Baidu para demostrar sofisticadas técnicas de inteligencia artificial para un dispositivo móvil o tableta, algo muy usado en estudios de cine y animación. Se basa en tecnología avanzada de aprendizaje que utiliza 72 puntos de características faciales, la definición de la boca, nariz, ojos, cejas, barbilla y mandíbula. El rostro se segmenta de acuerdo a los contornos faciales, la posición relativa y la forma de las principales características y expresiones faciales.

Taggar: Es una aplicación basada en realidad aumentada, que permite detectar rostros para añadirles accesorios que se acoplan en el rostro. 
Wikitude: Está basada en la tecnología de realidad aumentada y geolocalización. Con ella se puede obtener información sobre los lugares más significativos que tenemos a nuestro alrededor (museos, restaurantes, establecimientos...) gracias a las capas de información virtual que se superponen a la realidad.

Junaio: Es una aplicación con una funcionalidad similar a Wikitude, desarrollada por PublicAdCampaign y por The Heavy Projects, es un navegador de realidad aumentada para iPod Touch, iPhone e iPad que básicamente permite depurar y reemplazar las publicidades existentes en la vía pública por otros contenidos. Más información en web oficial: http://www.junaio.com y vídeotutorial: https://www.youtube. com/watch?v=9y6AMvhIg2c.

Goggles: Con esta aplicación podemos fotografiar objetos con nuestro móvil o tableta; y esos objetos será reconocidos para que Google nos muestre toda la información que tiene sobre ellos. Evidentemente esto funciona mejor con objetos muy reconocibles, como las portadas de libros, logos, cuadros, paisajes. También los desarrolladores de la aplicación han añadido soporte para tarjetas de visita, de tal manera que reconocerá teléfonos y demás información para añadir a nuestros contactos. Por otro lado, han incluido un modo de realidad aumentada que busca en tiempo real a medida que vamos moviendo la cámara y nos dice todo sobre los comercios que tengamos alrededor, utilizando también GPS y brújula para unos resultados más reales.

Aumentaty VSearch: Es una herramienta que nos permite, como publicadores, ofrecer contenido adicional basado en la tecnología de RA, vinculada a imágenes, para que se pueda consultar como usuario a través de la aplicación para dispositivos móviles. http://visualsearch. aumentaty.com.

Google Sky Map y Mapa Estelar: Son aplicaciones para apoyar el estudio de astronomía, principalmente para observar el espacio por las noches. El procedimiento consiste en enfocar la cámara del móvil o tableta hacia el cielo y el programa puede identificar estrellas, constelaciones, planetas y cuerpos celestes, y nos ofrece en vivo los datos. Las figuras 35 y 36 muestran capturas de pantalla de la aplicación para iOS Mapa Estelar. Sky Map también incorpora opciones de búsqueda de constelaciones.

3D Compass AR: Es una aplicación para situarnos y orientarnos en el espacio. A través de la cámara visualizamos una brújula digital y un pequeño mapa de Google Maps que se superponen sobre el con- texto real para indicar nuestra posición.

La Patena de Cástulo RA: Esta aplicación permite ver en realidad aumentada la patena de vidrio datada del s. IV d.C. y hallada en 2014 en el yacimiento arqueológico de Cástulo, a unos cinco kilómetros de Linares (Jaén). El estudiante puede observar con detalle su iconografía cristiana, una de las más antiguas y mejor conservadas de la península. Asimismo, puede verla desde todas sus perspectivas, ampliarla e incluso fotografiarse con ella. Más información: http://www.turismolinares.es/nueva-app-movil-la-patena-de-cristo-en-realidadaumentada Las figuras 18 y 19 muestran el marcador necesario y la patena de vidrio. 


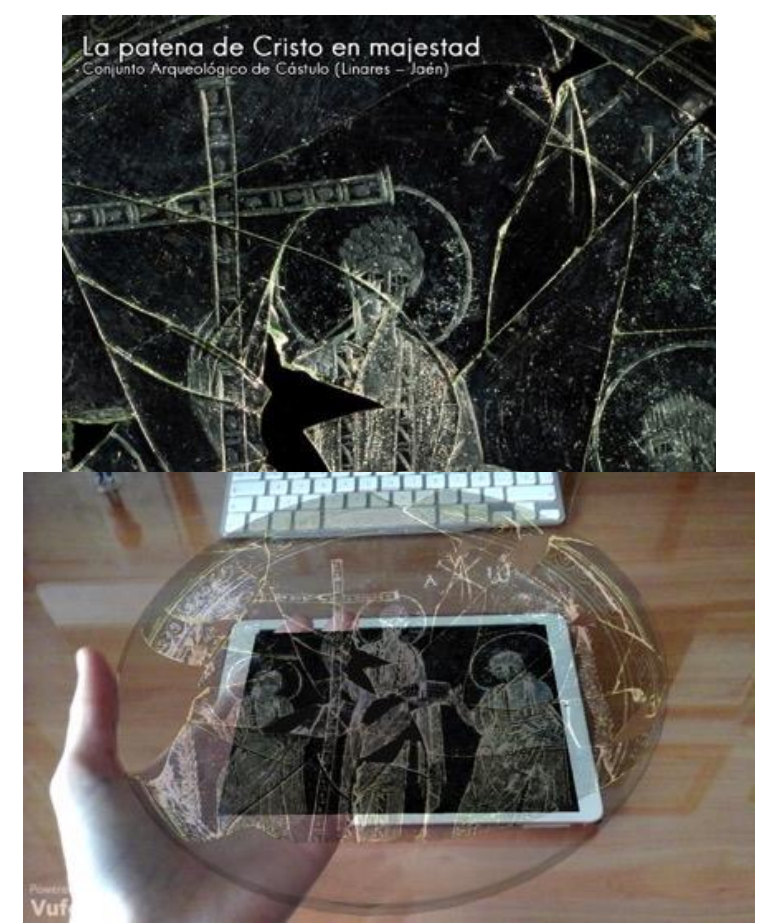

Figuras 18 y 19. Marcador y muestra de la patena de vidrio como modelo tridimensional.

AR Showcase: Nos ofrece una serie de modelos 3D que representan diversos objetos didácticos para materias de historia, arquitectura, medicina, biología, etc para su inserción y visualización en el contexto real.

Visuar: Es una aplicación para visualizar los elementos multimedia (imágenes, vídeos, audios y modelos 3D) que previamente, a través de la plataforma web Visuar, hemos generado y asociado a un marcador.

EspiraRA: aplicación de realidad Aumentada geolocalizada destinada específicamente al mundo educativo. Destinado a la creación de puntos (POIs) en el trazado de una ruta por parte del profesorado y del alumnado para el estudio sobre diversos temas, áreas y niveles educativos. Más información: http://aumenta.me

\subsection{Programas de RA para ordenador}

Aumentaty Author: Se trata de una programa para ordenador que permite la generación de contenidos de RA a partir de marcadores o fotografías, de los que se despliegan elementos virtuales tridimensionales ya creados previamente con el programa SketchUp, o bien obteniéndolos de su galería de modelos 3D. Aumentaty Viewer, es un programa complementario, también disponible como aplicación para móviles, que permite visualizar objetos tridimensionales mediante la cámara o webcam en diversos dispositivos. Más información: http://author.aumentaty.com. A continuación, en la figura 1 , se presenta un ejemplo de uso de la herramienta señalando y describiendo de forma detallada sus elementos. Como plantean Fombona, Pascual y Madeira (2012, p. 203) podemos observar cómo la RA amplía las imágenes de la realidad, a partir de su captura por la cámara de un equipo informático o dispositivo móvil avanzado que añade elementos virtuales para la creación de una realidad mixta a la que se le han sumado datos informáticos. 


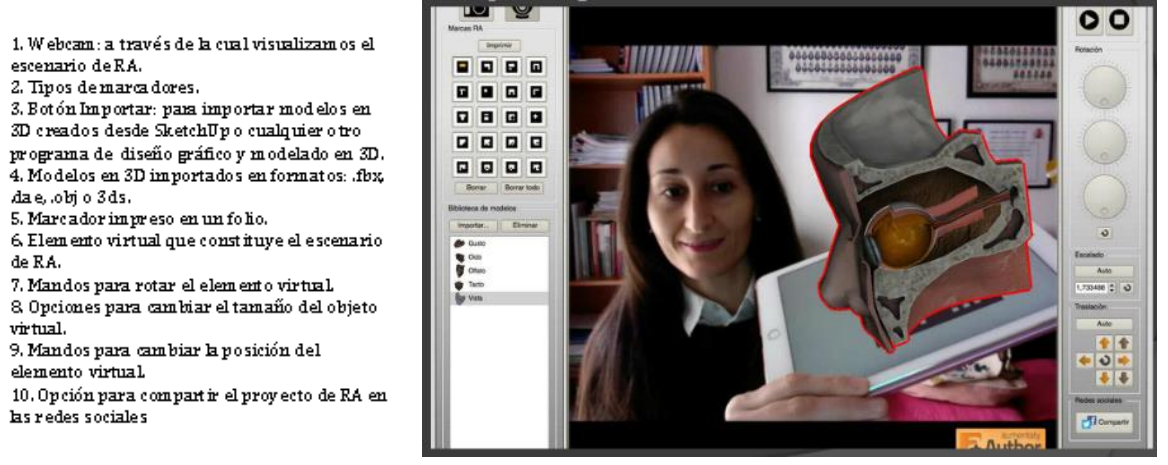

Figura 1. Herramienta Aumentaty Author y sus elementos

BuildAR: Permite crear escenarios en 3D de realidad aumentada de forma sencilla, similar al programa Aumentaty Author, nos ofrece 12 tipos de marcadores. Aunque podemos crear nuestros propios marcadores al mismo tiempo que se nos ofrece la posibilidad de superponer tres modelos de dimensión virtual en el mundo real a través de una webcam. Más información: http://www.buildar.co.nz

estARteco: Es el juego gratuito basado en la tecnología de realidad aumentada que permite al alumnado apreciar el valor de los ecosistemas y la complejidad de su equilibrio. Este transcurre en 4 fases de dificultad creciente, en las que tendrás que equilibrar la salud ecológica y los factores ambientales que conforman el entorno teniendo en cuenta además el impacto social y económico que provocan tus acciones. El programa ha sido desarrollado por el Área de Realidad Virtual y Realidad Aumentada de ITCL.

\subsection{Plataformas de aplicaciones web para crear y editar escenarios aumentados}

Augment: A través de esta plataforma se puede importar cualquier ar- chivo 3D en formato .dae, .kmz .obj, .fbx o .3ds, que podemos obte- ner de diversas galerías online como Warehouse 3D, entre otras. Una vez que descargamos el fichero del modelo tridimensional de dichas galerías, lo comprimimos en .zip y lo importamos en la plataforma Augment. Desde allí, a través del código Qr que se genera, lo escaneamos con la aplicación Móvil Augment, y obtenemos el modelo 3D para poderlo visualizar con detalle desde un marcador/tracker.

Aurasma Studio: Los creadores de la aplicación móvil Aurasma han puesto a disposición de los usuarios esta plataforma web a través de la cual se puede realizar una mayor variedad de acciones como crear auras (escenarios de RA) con modelos 3D para posteriormente importarlas a la aplicación de móvil. Además hace posible editar las auras ya creadas y añadir una o varias capas virtuales en diversos formatos de sonidos, imágenes, vídeos y gifs, las cuales se superponen a una imagen y estas pueden visualizarse tras ser escaneada con la aplicación móvil Aurasma.

LearnAR: Es otra aplicación para docentes y estudiantes que necesita de una cámara para su funcionamiento. En su web se puede acceder a actividades ya creadas para asignaturas tales como matemáticas, ciencias, anatomía, física, geometría, educación física e idiomas. Más información: http://www.learnar.org

ARCrowd: Aplicación online para la creación de entornos de RA personalizados. Más información en: http://arcrowd.com 
Bakia: Aplicación web para crear entornos de realidad aumentada a partir de modelos 3D en diversos formatos (.dae, .obj, .fbx o .3ds) que podemos generar y exportar desde el programa SketchUp o cualquier galería de modelos tridimensionales. Más información: http://www.bakia.co/experimenta.html

estARteco: Es una plataforma web que constituye una demo para la visualización de parte del juego gratuito basado en la tecnología de RA; el cual permite al alumnado apreciar el valor de los ecosistemas y la complejidad de su equilibrio. Con esta demo online se puede visualizar una pequeña parte del juego. La funcionalidad es muy limitada, pero gracias a ella se puede experimentar con la realidad aumentada para saber cómo funciona. En esta demo el alumnado podrá ver una parte de la ciudad y 3 factores: transporte, industria y consumo. Es necesaria una webcam conectada al ordenador y descargar los marcadores a través del enlace: http://www.estarteco.com/es/descarga, imprimirlos y enfocarlos con la cámara, y ver los objetos 3D del juego.

Visuar: Es una plataforma web en la cual podemos importar modelos 3D en formatos: .fbx, .obj, .dae e .ifc, imágenes, vídeos o audios y asociarlos al marcador genérico de la aplicación, y posteriormente, mediante la aplicación móvil Visuar, podemos visualizar dichos ele- mentos multimedia integrados en el contexto real. Más información: http://www.visuar.es

\section{Conclusiones.}

Con la implementación docente de estas herramientas emergentes se pretende avanzar en la incorporación de prácticas innovadoras en el aula sobre un andamiaje pedagógico y el diseño de actuaciones desde una perspectiva inclusiva partiendo de los principios de la creatividad y los modelos constructivista y conectivista para favorecer los aprendizajes. Aunque para ello, es necesario realizar previamente verdaderas adaptaciones de tipo metodológicas, didácticas, curriculares, organizativas, temporales, espaciales y formativas del profesorado.

Atendiendo a las demandas actuales y futuras de la sociedad del conocimiento y los nuevos modelos de aprendizaje del alumnado de la nueva era digital, se trata de aprovechar el potencial que nos ofrecen los medios tecnológicos bajo un planteamiento constructivista. $\mathrm{Y}$ en estos nuevos entornos de aprendizaje que se configuran con el uso de estas herramientas, el alumnado adquiere mayor protagonismo logrando un desarrollo de competencias basadas en el descubrimiento, la investigación, la exploración y la construcción del conocimiento de forma autónoma, colaborativa, creativa y reflexiva. Aunque lo más importante acerca de las características de estas herramientas y las propuestas de actividades que podemos plantear en el aula haciendo uso de esta tecnología, es el carácter global del aprendizaje que genera en un espacio mixto en el que se mezclan la virtualidad y la realidad, así como la idea de obtener una interactividad significativa y amplificada. Además, las ventajas de su aplicación a este ámbito del e-learning son muy diversas. En estos contextos de aprendizaje aumentados, se espera que los estudiantes estén más motivados para participar en el proceso de aprendizaje, al tratarse de actividades más interactivas, flexibles, dinámicas, versátiles y en las que el discente puede experimentar y manipular diversas situaciones (Leiva y Moreno, 2015; Moreno, Leiva y Ordóñez, 2015). 


\section{Referencias bibliográficas.}

Azuma, R. (1997). A Survey of Augmented Reality.Presence: Teleoperators and Virtual Environments, 6 (4), pp.: 355-385.

Basogain, X., Olabe, M., Espinosa, K., Rouèche, C.\& Olabe, J.C. (2007). Realidad Aumentada en la Educación: Una tecnología emergente. Comunicación presenta- da a Online Educa Madrid 2007: 7a Conferencia Internacional de la Educación y la Formación basada en las Tecnologías, Madrid.

Cabero, J. (2013). Ponencia: E-Learning 2.0. 3er Congreso Internacional sobre Buenas Prácticas con TIC en la Investigación y la Docencia. Universidad de Málaga. 23-25 de octubre.

Cabero, J. Y García, F. (2016). Realidad Aumentada. Tecnología para la formación. Madrid: Síntesis.

Cabero J., Leiva J.J., Moreno N.M., Barroso J. y López-Meneses, E. (2016). Realidad Aumentada y Educación. Innovación en contextos formativos. Barcelona: Octaedro.

Cobo, C. y Moravec, J.W. (2011). Aprendizaje invisible. Hacia una nueva ecología de la educación. Col.lecció Transmedia XXI. Laboratori de Mitjans Interactius.

Barcelona: Univesitat de Barcelona. Recuperado de: http://www.aprendizajeinvisible.com/es/

De Pedro, J. (2011). Realidad Aumentada: un nuevo paradigma en la educación superior. En E. Campo, M. García, E. Meziat \& L. Bengochea (eds.). Educación y sociedad. (pp. 300-307). Chile: Universidad La Serena.

Di Serio, Á., Ibáñez, M. B., y Kloos, C. D. (2013). Impact of an augmented reality system on students' motivation for a visual art course. Computers y Education, 68, pp.: 586-596.

Dunleavy, M. y Dede, C. (2014). Augmented reality teaching and learning. Handbook of research on educational communications and technology. New York: Springer.

Escuny, C., González, J., Lleixá, M. y Gisbert, M. (2011). Actitudes y expectativas del uso educativo de las redes sociales en los alumnos universitarios, RUSC, 8(1), pp.171185.

Fombona, J., Pascual, M.A. \& Madeira, M.F. (2012). Realidad Aumentada, una evolución de las aplicaciones de los dispositivos móviles. Píxel-Bit. Revista de Medios y Educación, 41, pp.: 197-210.

García, A. (2008). "Las redes sociales como herramientas para el aprendizaje colaborativo: una experiencia con Facebook". En Actas del XIII Congreso Internacional en Tecnologías para la Educación y el Conocimiento: la Web 2.0. Madrid: UNED.

Gómez, M. (2013). Educación Aumentada con Realidad Aumentada. En 3er Congreso Internacional sobre Buenas Prácticas con TIC en la Investigación y la Docencia. Universidad de Málaga. 23-25 de octubre. 
Horizon Report NMC (2012) (2013) (2014) (2015) Higher Education Edition. Recuperado de: http://www.nmc.org/publication/nmc-horizon-report-2015-highereducation-edition

Kato, H. (2010). Return to the origin of Augmented Reality [Archivo de vídeo]. Presentation at IEEE International Symposium on Mixed and Augmented Reality 2010 (Seoul, Korea). Panel discussion: «The Future of ISMAR: Converging Science, Business, and Art» (organized by Henry Fuchs and Christian Sandor). Recuperado de: http://www.youtube.com/watch ?v=b33eqcVz7X8

Leiva, J.J y Moreno, N.M. (2015). Tecnologías de Geolocalización y Realidad Aumentada en Contextos Educativos: Experiencias y Herramientas Didácticas, DIM, 31, págs.:1-15. Recuperado de: http://dialnet.unirioja.es/servlet/articulo?codigo $=5081652$ \&orden $=0 \& i n f o=l i n k$

Leiva, J. (2015). Las esencias de la Educación Intercultural. Archidona (Málaga): Aljibe.

Moreno, N.M., Leiva, J.J. y Ordóñez, E. (2015). La realidad aumentada como factor de calidad e innovación educativa. En J.L. Sarasola Sánchez-Serrano; L. Molona García; M.I. Hernández Romero; N.M. Moreno Martínez y E. López Meneses (Dirs.), I Seminario Internacional Hispano-Mexicano de Formación, Investigación e Innovación Universitaria 9 y 10 de Junio de 2015. Madrid: Afoe.

Ortiz, C. (2011). Realidad Aumentada en Medicina. Revista Colombiana de Cardiología, 18 (1), págs.: 4-7.

Pérez, A.I. (2012). Educarse en la era digital. Madrid: Morata.

Reig, D. (2012). Horizont Report 2012 a 2017: Tecnologías y aprendizaje en los próximos años. Blog El Caparazón. Recuperado de:

http://www.dreig.eu/caparazon/2012/02/05/horizon-report-2012

Reig, D. (2013). Los significados educativos y sociales de la revolución móvil. Mobile World Capital Barcelona. Recuperado de:

http://mobileworldcapital.com/es/articulo/183

Reig, D. y Vílchez, L.F. (2013). Los jóvenes en la era de la hiperconectividad: tendencias, claves y miradas. Madrid: Fundación Telefónica. Recuperado de: http://www.fundacion.telefonica.com/es/arte_cultura/publicaciones/detalle/182

Reinoso, R. (2012). Posibilidades de la realidad aumentada en educación. En J. Hernández, M. Pennesi, D. Sobrino \& A. Vázquez (Coords). Tendencias emergentes en educación con TIC. (pp.357-400). Barcelona: Editorial espiral. 